\title{
Prévoir l'évolution des mouvements de terrain
}

\author{
Forcasting of landslide evolution
}

\author{
J.-L. FAVRE* \\ Ecole Centrale de Paris, Laboratoire de mécanique des sols, structures et matériaux, \\ CNRS, URA 850 \\ E. GERVREAU** et J.-L. DURVILLE** \\ Laboratoire Central des Ponts et Chaussées. \\ Division géologie de l'aménagement et de l'environnement
}

Rev. Franç. Géotech. n 59, pp. 65-73 (avril 1992)

\section{Résumé}

Le développement récent d'instrumentations permettant un suivi cinématique des glissements de terrain en continu a favorisé la mise au point de quelques méthodes de prévisions temporelles des mouvements de versants.

Des modèles univariés sont d'abord présentés : ils ne nécessitent que la connaissance d'un paramètre cinématique (déplacement ou vitesse) et permettent, dans certains cas, de prévoir l'évolution des mouvements, en particulier une éventuelle rupture.

Dans un deuxième temps est proposé un modèle explicatif orienté sur la cause hydraulique, capable de prévoir l'évolution future proche des vitesses de déplacement des versants sensibles aux variations climatiques.

\footnotetext{
Abstract

Recent progresses in monitoring of landslides allow to build up some movement forecasting techniques and methods for predicting the failure time of slope.

At first, we present univaried models which need only a time series of displacement data and permit, in some case, foreseeing movement or predicting the failure time.

In a second time an explicative movement model based on the hydraulic cause is proposed. It is able to forecast near future displacement evolution of a landslide influenced by rainfall.
} 


\section{POSITION DES PROBLÈMES}

Face aux versants naturels en mouvement les problèmes posés aux géotechniciens sont généralement formulés ainsi :

- prévoir l'évolution des déplacements (vitesses) au cours du temps;

- évaluer le risque de rupture catastrophique à une date future qu'il faut estimer:

- connaître l'effet de mesures confortatives sur le champ des vitesses et, en particulier, assurer la stabilisation ;

- calculer les sollicitations induites par les masses en mouvement sur les constructions existantes.

Sur ces points les méthodes d'analyse font cruellement défaut, même si quelques solutions sont proposées pour des cas particuliers. Ceci tient sans doute d'abord au développement relativement récent d'instrumentations propres à assurer un suivi cinématique des glissements, mais, même sur des sites instrumentés, les méthodes de prévision sont encore loin d'être totalement satisfaisantes.

L'objectif de la présente étude est de tenter d'apporter des éléments de réponses aux deux questions suivantes :

- quelle sera, au cours du temps futur proche, l'évolution des déplacements d'un versant en mouvement? - une brutale accélération du phénomène est-elle possible ? si oui, à quelle date la rupture est-elle plus probable?

Une démarche purement phénoménologique nous est apparue très vite mal adaptée, car il est très difficile sur un versant naturel d'identifier et de modéliser l'ensemble des phénomènes en présence, surtout lorsqu'il s'agit d'un système qui évolue rapidement au cours du temps.

Nous avons retenu une démarche plus empirique, en utilisant des traitements de signaux temporels qui sont bien adaptés à la modélisation de systèmes évolutifs. Il s'agit d'une approche qui peut surprendre, car généralement les ingénieurs modélisent en termes de contraintes et de déformations. Nous avons cependant estimé qu'elle peut apporter des solutions intéressantes et nous allons tenter ici d'en mettre quelques unes en lumière.

\section{LES MOUVEMENTS DE TERRAIN}

Le vocable "mouvement de terrain", dans son acception courante regroupe des phénomènes d'instabilité de pentes, naturelles ou non, très variés tant en volume qu'en nature de matériau ou en mécanisme mis en jeu (fig. 1a à 1e).

En France tous ces types de mouvement sont rencontrés, mais les travaux présentés dans cet article concernent plus particulièrement les glissements plans et circulaires de pentes naturelles en mouvement. L'agent principal de ces phénomènes est la pesanteur; les sollicitations au sens d'une analyse mécanique varient donc relativement peu. Ce sont de faibles variations de contraintes et de propriétés mécaniques au niveau de la surface de rupture qui expliquent les mouvements importants de certains versants instables.

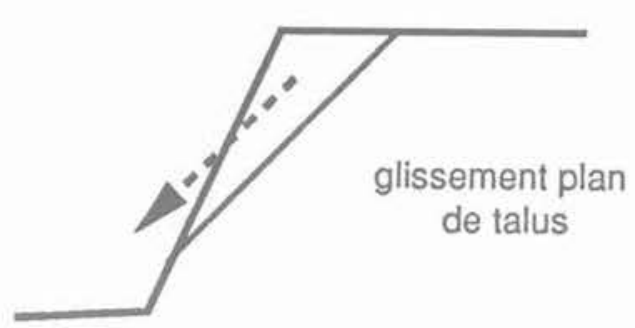

a
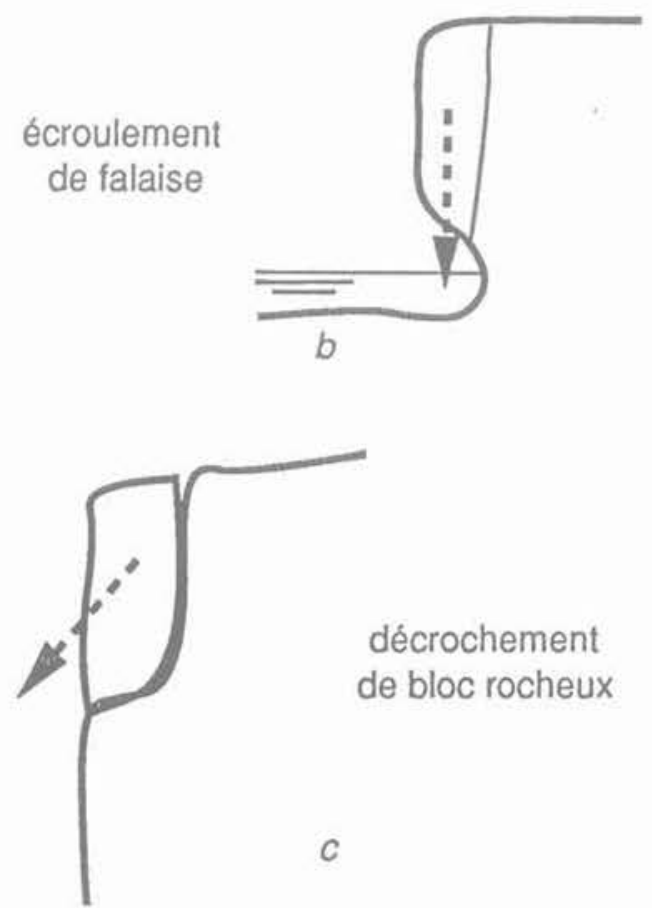

décrochement de bloc rocheux
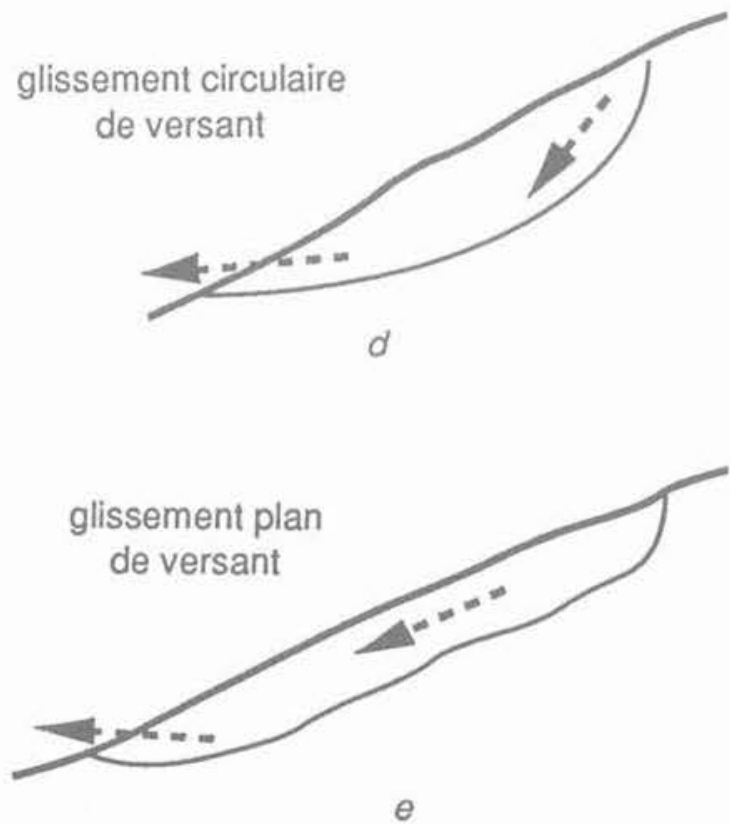

Fig. 1. - Quelques exemples de mouvements de versants. Fig. 1. - Some examples of landslides. 


\section{3. ÉVOLUTION DES MOUVEMENTS DE TERRAINS}

D'une manière générale la dynamique d'un mouve. ment de versant peut être décomposée en cinq phases (fig. 2).

Phase 1 : déplacements très lents n'entrainant pas de désordres importants; les vitesses de déplacement observées sont presque constantes.

Phase 2: apparition de mouvements plus importants ; la stabilité est mise en cause.

Phase 3: rupture proprement dite.

Phase 4 : les vitesses diminuent, le versant s'achemine vers une position d'équilibre.

Phase 5: le versant est stabilisé pour une durée indéterminée.

Dans la pratique il n'est pas toujours facile de savoir dans quelle phase du mouvement on se trouve à un instant donné sur un versant.

temps

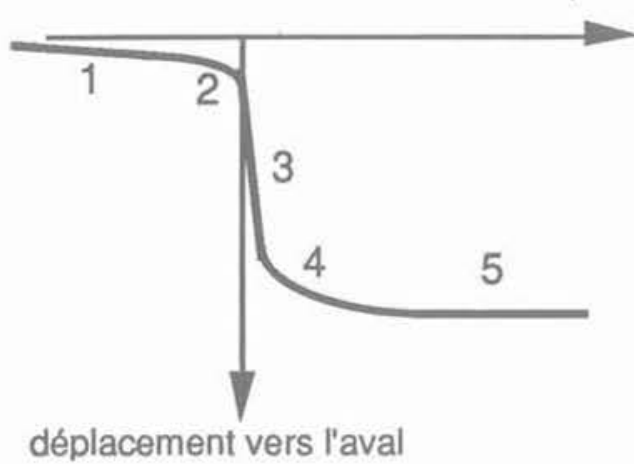

Fig. 2. - Schéma d'évolution des déplacements d'un versant instable.

Fig. 2. - Diagrarn of displacement evolution of a moving slop.
Les versants naturels en mouvement posent souvent des problèmes de sécurité des biens et des personnes. Le site le plus spectaculaire à cet égard actuellement est celui de La Clapière à Saint-Etienne-deTinée (Alpes-Maritimes) (fig. 3).

Cinquante millions de $\mathrm{m}^{3}$ de roches gneissiques (voir figure 4) se déplacent vers le fond de la vallée avec des vitesses qui ont atteint $100 \mathrm{~mm} / \mathrm{j}$ en 1987. Actuellement est observée une extension de l'instabi-

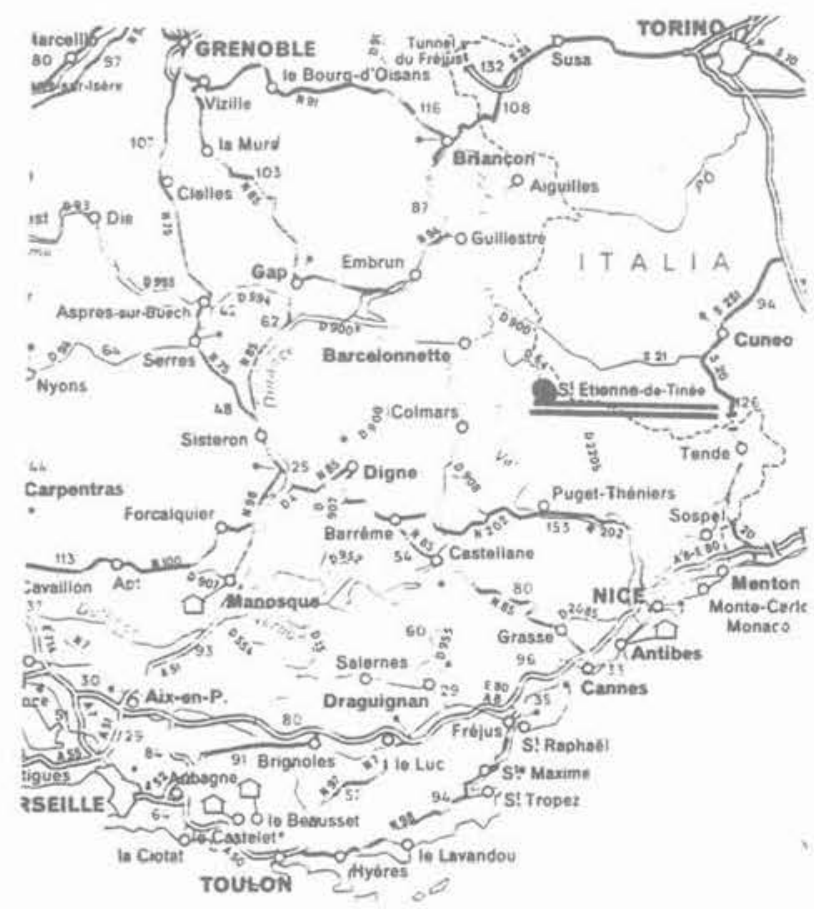

Fig. 3. - Localisation du site de La Clapière à Saint-Etienne-de-Tinée.

Fig. 3. - Location of the La Clapière landslide near Saint-Etienne-de-Tinée.

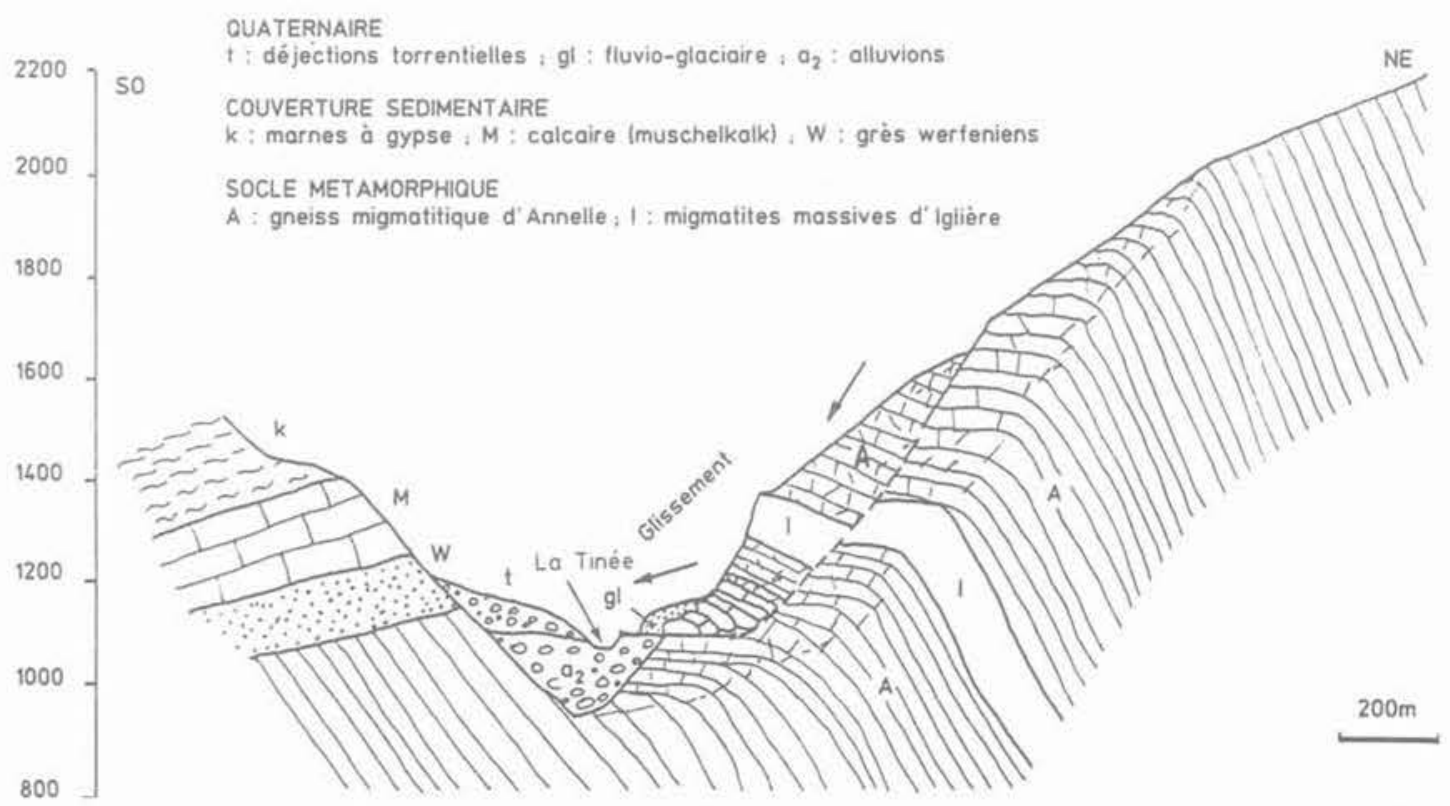

Fig. 4. - Coupe géologique à travers la vallée de la Tinée et le glissement. (Extrait de J.-P. FOLLACCI, 1987). Fig. 4. - Geological cross-section of La Clapière landslide. (From J.-P. FOLLACCI, 1987). 
lité par l'amont avec des vitesses de déplacement de l'ordre de $20 \mathrm{~mm} / \mathrm{j}$ alors que celles du glissement principal sont redescendues à $10 \mathrm{~mm} / \mathrm{j}$ environ.

D'après FOLLACCl (1987), un fauchage est apparu lors de la déglaciation du quaternaire, il a eu pour effet de fracturer la masse du gneiss, et d'accroître la porosité et la perméabilité du versant. Depuis une cinquante d'années, la surface de rupture actuelle s'est formée progressivement, et les déplacements cumulés atteignent aujourd'hui $80 \mathrm{~m}$ par endroit.

Sur la courbe d'évolution des mouvements depuis 1983 (fig. 5), on observe le paroxysme de 1987 et une composante périodique des vitesses qui correspond à des fluctuations saisonnières de conditions climatiques.

Le risque de rupture était jugé très élevé en 1987 ; malgré le ralentissement observé depuis, ce risque ne peut pas être écarté avec certitude aujourd'hui.

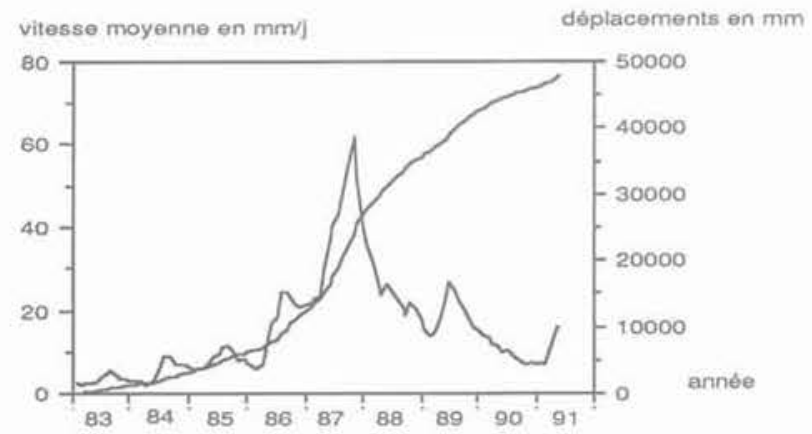

Fig. 5. - Evolution des déplacements

et des vitesses de déplacement de janvier 1983 à mai 1991 du glissement principal de La Clapière.

Fig. 5. - Displacement and rate of displacement evolution from january 1983 to may 1991 of La Clapière landslide

\section{LA PRÉVISION TEMPORELLE DU MOUVEMENT}

Certains mouvements de versant sont assez réguliers pour que leur observation pendant un intervalle de temps suffisant donne sur le futur une information assez complète. On procède alors à des techniques qui s'apparentent à des traitements de signaux univariés, donc sans tenir compte explicitement des facteurs de déclenchement ou d'évolution. Cette approche purement empirique donne dans certains cas de bons résultats en terme de prévision comme nous le verrons au paragraphe 4.1.

Cependant la plupart des mouvements sont sensibles à des causes extérieures diverses, et la prévision temporelle est basée sur l'existence de relations plus ou moins directes de cause à effet entre ces facteurs de déclenchement, eux-mêmes prévisibles ou non, et les mouvements (fig. 6)

Les facteurs externes qui gouvernent les mouvements sont nombreux et, parfois, difficiles à identifier ; très souvent la quantification de l'influence de chaque facteur pose des problèmes. Une modélisation de

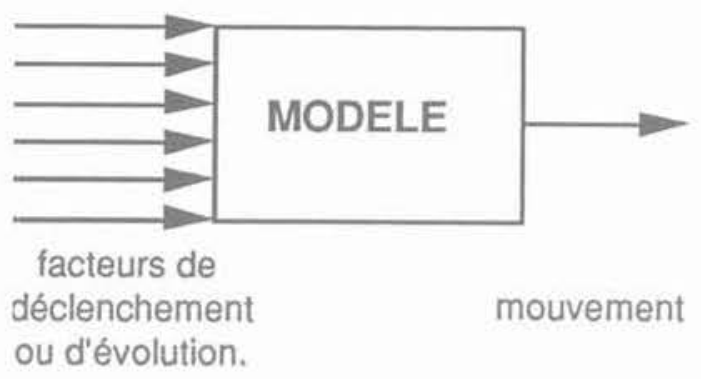

Fig. 6. - Schéma d'un modèle explicatif.

Fig. 6. - Diagram of explicative model.

l'ensemble des phénomènes en jeu n'est donc pas opérationnelle. De plus, les informations sur les versants naturels instables sont relativement peu nombreuses car coûteuses et même parfois techniquement inacessibles.

Nous proposons une solution dite pseudo-empirique qui constitue un compromis entre la volonté d'effectuer des analyses précises et fines et le désir d'obtenir des résultats directement exploitables dans un cadre décisionnel.

Ceci nous amène à parler, dans ce cas intermédiaire, de a boîte grise * qui sera décrite au paragraphe 4.2.

\subsection{Modèles univariés}

Les modèles de prévision univariés ou autoprogressifs admettent implicitement que le temps seul constitue lindex d'évolution prépondérant. II s'agit de modèles de mise en cuvre économique car ils ne nécessitent la connaissance au cours du temps que d'un paramètre décrivant le mouvement, mais ce sont aussi les moins riches d'un point de vue analytique. Leur principe consiste à tirer de l'évolution passée des mouvements l'information pour prévoir l'évolution future. A court terme, c'est-à-dire à des horizons de quelques jours il est possible par ces méthodes d'obtenir de bonnes prévisions si le système mécanique que constitue le versant évolue de façon continue ou régulière, c'est-à-dire si les sollicitations et les propriétés mécaniques ne varient pas de façon erratique dans le temps. L'exploitation de ces modèles consistera simplement à faire de l'ajustement plus ou moins sophistiqué (modélisation) puis de l'extrapolation (prévision).

\subsubsection{Modèles de prévision d'évolution des mouvements}

Prenons à titre d'exemple le glissement du Vajont (Mont Toc, Italie) d'un volume de 250 millions de $\mathrm{m}^{3}$ de roche fracturée marno-calcaire dont la rupture brutale a provoqué indirectement 2000 morts le 9 octobre 1963 (voir J.A. CORBYN, 1982). La série des déplacements durant les huit semaines précédant la rupture a fait l'objet d'un ajustement polynomial de degré 3 (fig. 7), et des extrapolations ont été effectuées à partir du 1er octobre 1963.

On constate que les prévisions de déplacements sont inférieures aux observations ; cette divergence positive peut être interprétée de trois façons: 


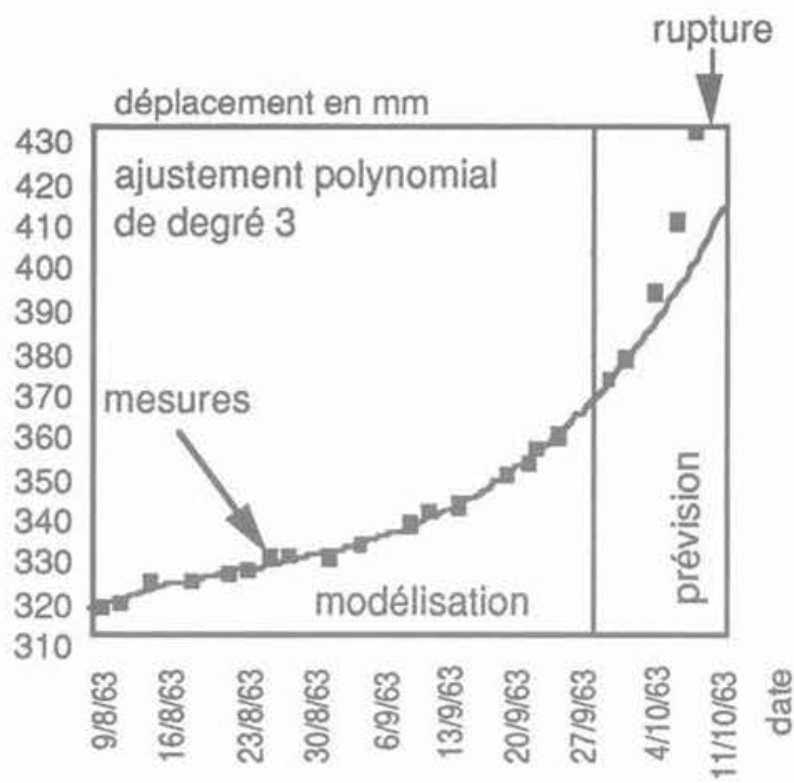

Fig. 7. - Evolution des déplacements du glissement du Vajont d'août 1963 jusqu'à la rupture.

Fig. 7 . - Displacement evolution from 9 august 1963 to 9 october 1963 , date of failure of the Vajont.

- l'équation ajustée est mal choisie (courbure insuffisante) :

- le modèle univarié ne convient pas, et des facteurs d'évolution externes doivent être pris en compte :

- Pévolution des mouvements laisse entrevoir une rupture imminente.

Dans ce cas les trois interprétations sont possibles, mais la deuxième est peut-être la meilleure. En effet, sur ce versant baigné en pied par une retenue d'eau. une vidange rapide a été décidée, de façon probablement inconsidérée, dès l'apparition de mouvements importants, ce qui a peut être précipité la rupture.

Il existe d'autres techniques de prévision plus performantes comme le lissage exponentiel double: pour des séries qui présentent une tendance linéaire croissante, on suppose que les observations peuvent être ajustées au voisinage de $\mathrm{t}=\mathrm{T}$ par la droite $\mathrm{y}(\mathrm{T}+$ $t)=a_{0}(T)+a_{1}(T) \cdot(t-T)$. La détermination des paramètres $a_{0}(T)$ et $a_{1}(T)$ se fait par calage sur les observations précédant le temps $T$, avec une pondération à l'aide d'un paramètre $\alpha$ qui favorise les mesures les plus récentes.

Dans la pratique l'utilisation de la méthodes consiste à caler le paramètre $\alpha$ à partir d'une série d'observations représentatives, puis à calculer les prévisions à l'horizon choisi lors du calage, à chaque nouvelle observation.

Nous avons testé cette méthode sur le glissement de Séchilienne (ANTOINE et al., 1987) (fig. 8). Pour la période relativement stable retenue ici on constate que la méthode a permis de dégager de bonnes prévisions ; en revanche, appliquée dans des périodes plus perturbées, elle ne donne pas de bons résultats.

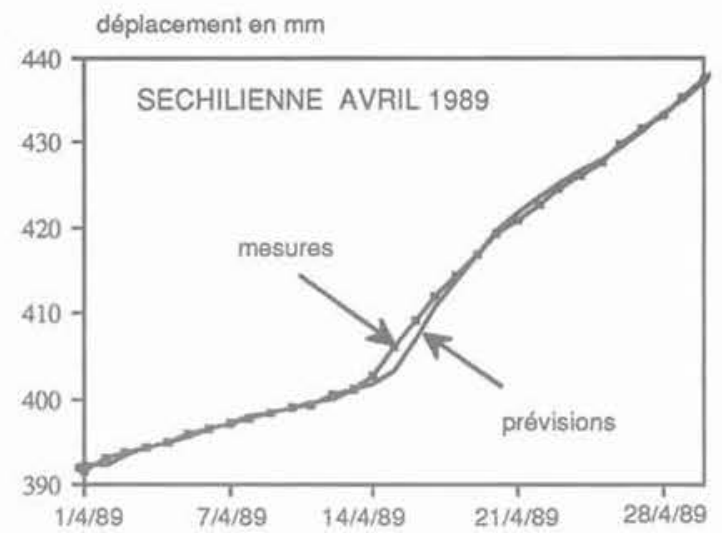

date

Fig. 8. - Prévision à I'horizon un jour des déplacements du glissement de Séchilienne par lissage exponentiel double.

Fig. 8. - One day horizon forecasts of displacement at Sechilienne landslide with dauble exponential smooth method.

\subsubsection{Modèles de prévision de date de rupture}

Nous présentons deux méthodes de prévision de date de rupture qui admettent pour hypothèse que la courbe de déplacement ou de vitesse en fonction du temps avoisine une asymptote verticale à l'approche de la rupture (B. VOIGHT, 1989).

La méthode de M. SAITO (1969) découle analytiquement de la relation suivante :

$$
\log \left(t_{r}-t\right)=C-D \log (d \epsilon / d t)
$$

où :

$\epsilon=\Delta \mathrm{l} / \mathrm{l}_{\mathrm{o}}$

$\Delta \mathrm{l}$ : déplacement entre deux points de mesures;

$\mathrm{I}_{0}$ : distance initiale entre deux points de mesures : $\mathrm{d} \epsilon / \mathrm{dt}$ : vitesse de déformation dans la phase finale ;

$t_{\mathrm{r}}$ : temps où se produit la rupture ;

C et D: constantes.

Une construction graphique élégante (fig. 9) permet de prévoir la date de rupture $t_{\mathrm{T}}$ à partir du graphe représentant l'évolution des déplacements en fonction du temps.

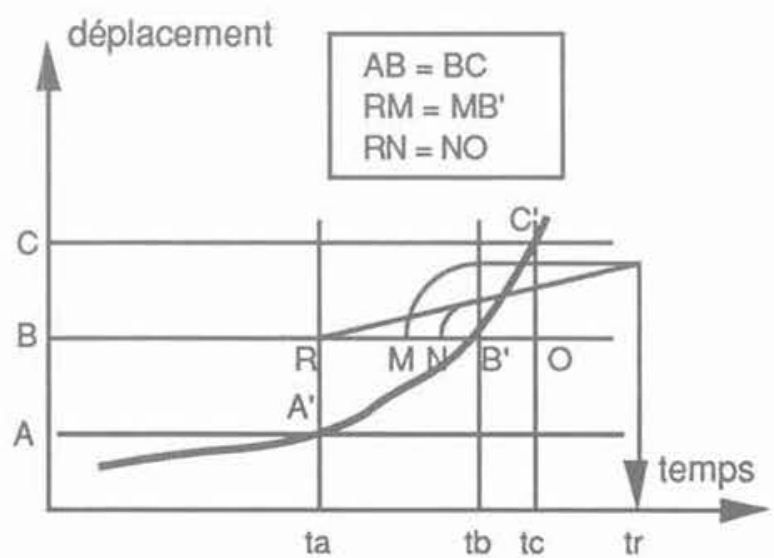

Fig. 9. - Construction graphique proposée par M. SAITO pour prévoir la date de rupture.

Fig. 9. - M SAITO graphical construction to predict the time failure of landslide. 
La méthode d'ASAOKA (1978) initialement énoncée pour les tassements maximaux dus à la consolidation des sols a été proposée par C. AZIMI et al. (1986) pour prévoir la date de rupture de glissement de terrain.

Le principe est de découper l'échelle des mouvements $\mathrm{Y}$ en intervalles égaux $\Delta \mathrm{Y}$ correspondant à des temps successifs croissant $t_{1}$ dont les intervalles $\Delta t_{1}=t_{1}-$ $t_{1}-1$ tendent vers 0 si $Y$ tend vers l'infini (fig. 10).

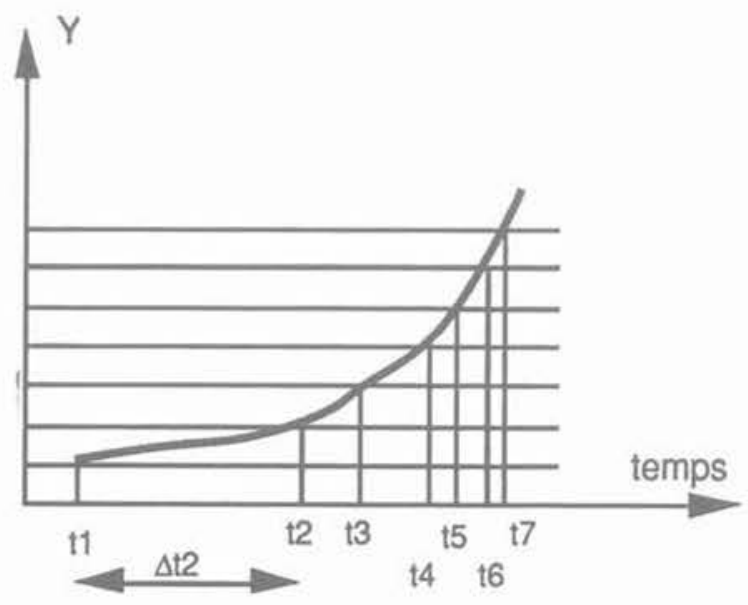

Fig. 10. - Découpage d'une courbe schématique de déplacement en intervalles $\Delta Y$ égaux.

Fig. 10. - Schematical displacement curve before failure.

La prévision du temps de rupture $\mathrm{t}_{\mathrm{r}}$ est l'intersection de la courbe $t-\Delta t$ avec l'axe $\Delta t=0$ (fig. 11).

Les deux méthodes présentées ont été testées sur une dizaine de cas de rupture de versants (fig. $12, \mathrm{j}$ : jour de la rupture). Nous constatons que les prévisions sont d'autant plus satisfaisantes que l'on est proche

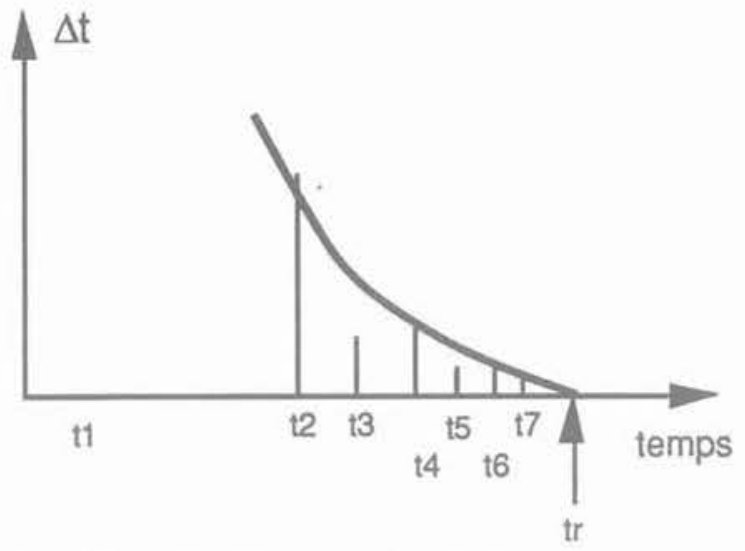

Fig. 11. - Variation de $\Delta t$ permettant une prévision de rupture.

Fig. 11. - $\Delta$ t evolution and time fallure predicting.

de la date de rupture ; un suivi en continu paraît donc souhaitable.

\subsection{Modèle multivarié orienté sur la cause hydraulique}

Le modèle proposé est orienté sur la cause hydraulique qui est bien souvent prépondérante. L'eau agit sur la stabilité des versants, principalement par les variations de pressions interstitielles (piézométrie) au niveau de la surface de rupture.

La première phase de la modélisation consiste à déterminer la variable d'entrée, c'est-à-dire les apports d'eau dans le sol, à partir des précipitations.

Nous pouvons ensuite quantifier les liaisions apports d'eau-piézométrie, puis piézométrie-mouvement, pour constituer un modèle de comportement orienté sur la cause hydraulique. Nous présentons ci-dessous un exemple, baptisé "modèle à un réservoir ».

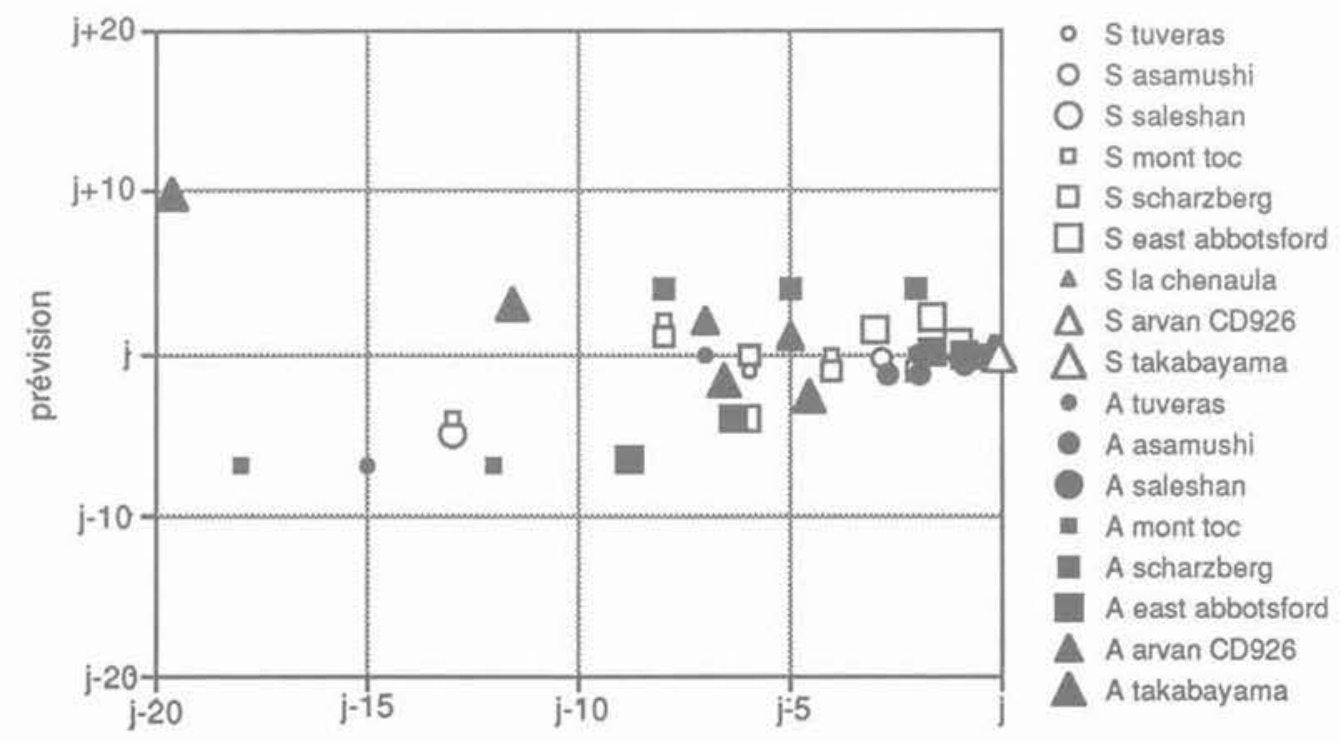

date de prévision (date de la dernière mesure prise en compte par rapport à la date de rupture j)

Fig. 12. - Résultats obtenus par les méthodes de M. SAITO et d'ASAOKA sur quelques cas de rupture. Fig. 12. - results of M. SAITO and ASAOKA methods failure cases histories. 


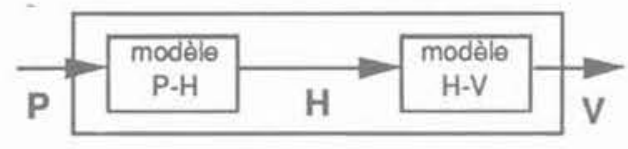

Fig. 13. - Schéma du modèle explicatif orienté sur la cause hydraulique.

Fig. 13. - Diagram of explicative model based on the hydraulic cause.

$P$ : Apports d'eau en surface;

$\mathrm{H}$; Paramètre piézométrique ;

$\mathrm{V}$ : Vitesse de déplacement.

La première partie du modèle permet de quantifier la liaison qui existe dans les versants entre les apports d'eau en surface et les niveaux piézométriques.

Il s'agit d'un modèle hydraulique simple : réservoir à surface libre, muni à sa base d'un orifice de vidange et alimenté par sa surface, ce qui nous permet de reconstituer un paramètre piézométrique.

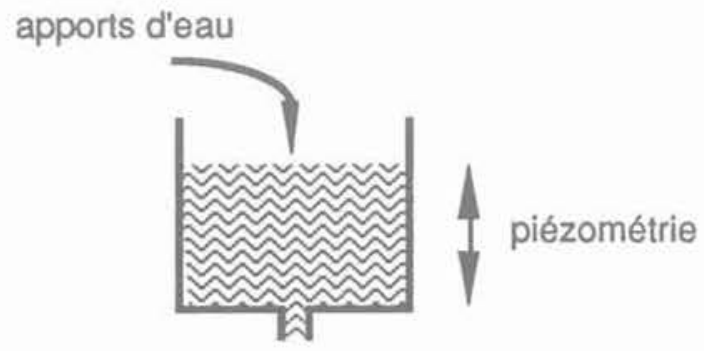

Fig. 14. - Schéma du modèle à un réservoir. Fig. 14. - Diagram of reservoir model.

Le calcul itératif de la piézométrie $\mathrm{H}$ au temps $\mathrm{j}+$ 1 à partir des apports d'eau $\mathrm{P}$ enregistrés durant l'intervalle de temps unité et de la valeur piézométrique précédente $\mathrm{H}$ (i) est effectué grâce à la formule suivante :

$$
\mathrm{H}(\mathrm{i}+1)=(\sqrt{\mathrm{H}(\mathrm{i})}-\beta)^{2}+\alpha \mathrm{P}(\mathrm{i})
$$

$\beta$ et $\alpha$ sont des paramètres de calage, respectivement coefficient de décharge et de recharge de nappe.
Utilisée isolément cette partie du modèle permet de prévoir les variations piézométriques d'un terrain avec des bons résultats.

La deuxième partie consiste à modéliser la liaison entre la piézométrie et la vitesse de déplacement de la masse en mouvement.

La relation retenue est linéaire, ce qui, d'un point de vue théorique peut correspondre à un comportement visqueux, les variations de niveau piézométrique se traduisant par des modifications d'état de contrainte au niveau de la surface de rupture.

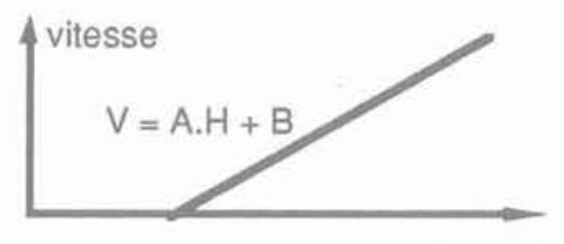

Piézométrie

Fig. 15. - Modèle H-V.

Fig. 15 . - Diagram of $\mathrm{H}-\mathrm{V}$ model.

L'utilisation du modèle global ne nécessite pas la connaissance des niveaux piézométriques dans les versants. $\mathrm{H}$ n'est alors qu'un paramètre intermédiaire dont les variations sont supposées proportionnelles à la piézométrie réelle. Le calage des paramètres du modèle est effectué à partir des séries connues "Apports d'eau au massif * et « Vitesse \#. Les valeurs des paramètres du modèle sont déterminées de manière à avoir la meilleure corrélation linéaire possible entre $H(t)$ et $V(t)$.

Pour le glissement de La Clapière sur la période mai 1990-octobre 1990 nous avons effectué un calage des paramètres du modèle global en retenant un temps de réponse du système égal à un jour (fig. 16 et 17).

Il convient ici de signaler les difficultés liées à la connaissance des apports d'eau au massif. En effet, ce sont les eaux infiltrées $P$ qui conditionnent les niveaux de nappes et donc les mouvements, et nous mesu-

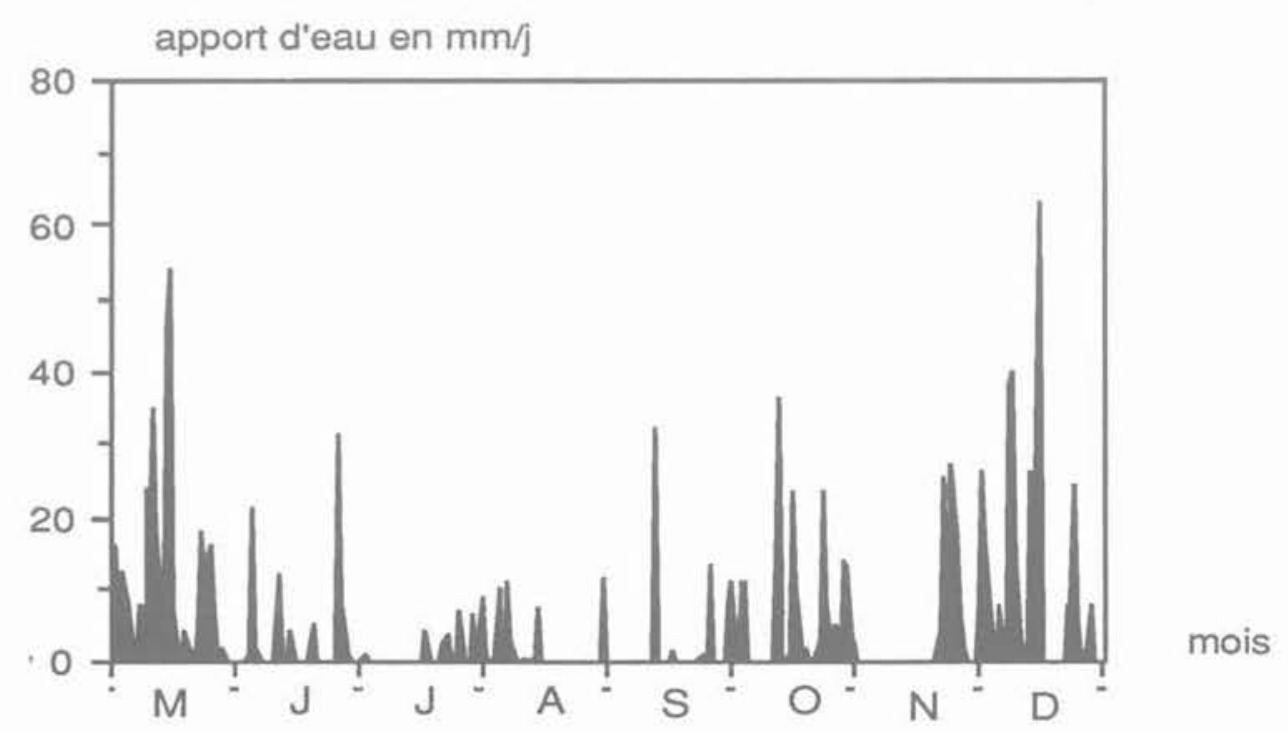

Fig. 16. - Apports d'eau au versant de La Clapière de mai 1990 á décembre 1990.

Fig. 16. - Water input at La Clapière from may 1990 to december 1990. 


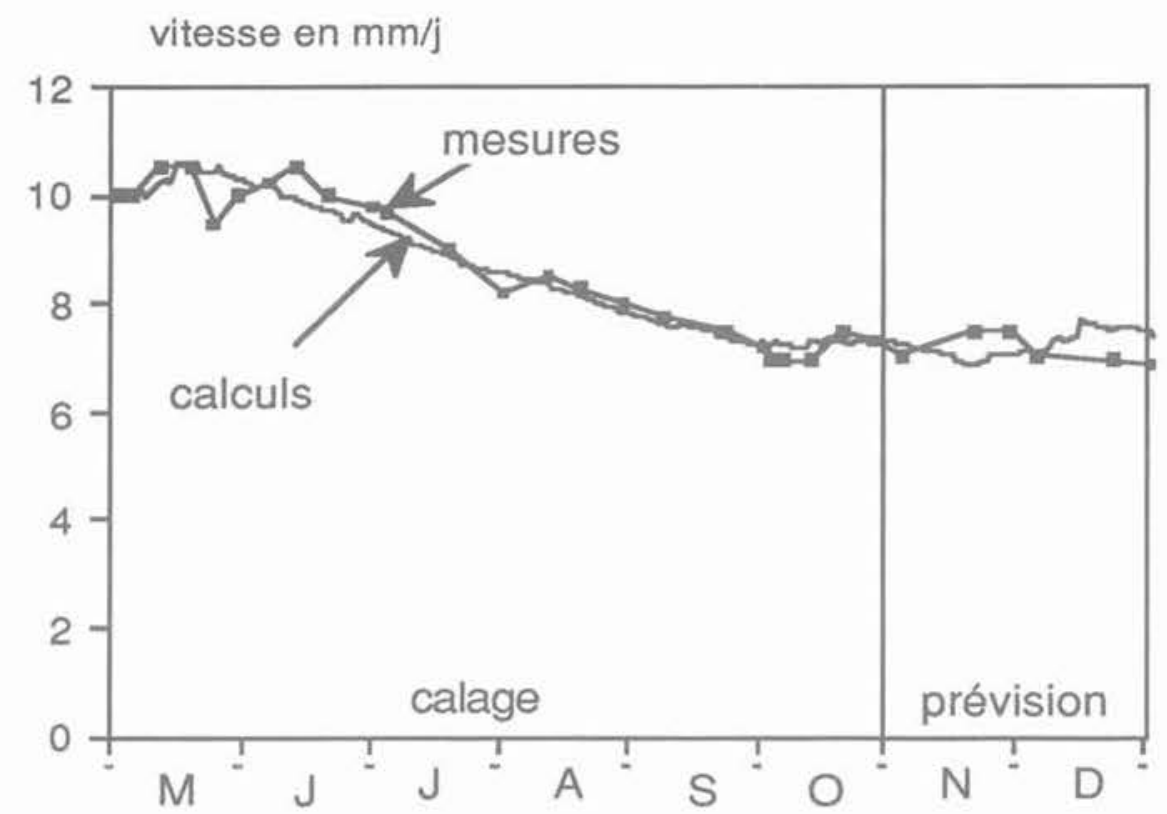

mois

Fig. 17. - Vitesses mesurées et vitesses calculées du glissement de La Clapière de mai 1990 à décembre 1990.

Fig. 17. - Comparaison between observed and computed rate of displacement at La Clapiére from may 1990 to decembre 1990.

rons en surface des précipitations; ceci amène donc une connaissance indirecte et parfois imprécise de la quantité d'eau infiltrée dans le massif avec laquelle il faut bien caler le modèle (pour le versant de La Clapière nous avons retenu $\mathrm{P}=$ Pluies + fusion de neige).

Le coefficient de corrélation de calage est satisfaisant (proche de l'unité). Pour les mois de novembre et décembre 1990 , nous avons effectué des prévisions (à l'horizon un jour) à partir des apports d'eau quotidiens.

La confrontation des prévisions aux observations sur ces deux mois montre une bonne coïncidence (fig. 17) mais durant cette période les apports d'eau ont été faibles.
Le site de La Clapière est en zone montagneuse (altitude : $1100 \mathrm{~m}-1700 \mathrm{~m}$ ), par conséquent le versant est enneigé en période hivernale, ce qui réduit bien sûr les apports durant cette saison. En revanche, au printemps, il y a fusion du manteau neigeux, ce qui a pour conséquence une forte alimentation des nappes entraînant des accélérations du mouvement. De décembre 1990 à mai 1991, nous avons effectué un calage du modèle avec également une bonne corrélation (fig. 18 et 19).

Pour d'autres périodes, le calage et la prévision sont moins bons ; ceci s'explique généralement par une mauvaise estimation des apports d'eau au massif.

Le système mécanique modélisé évolue plus ou moins rapidement au cours du temps, cela se traduit au

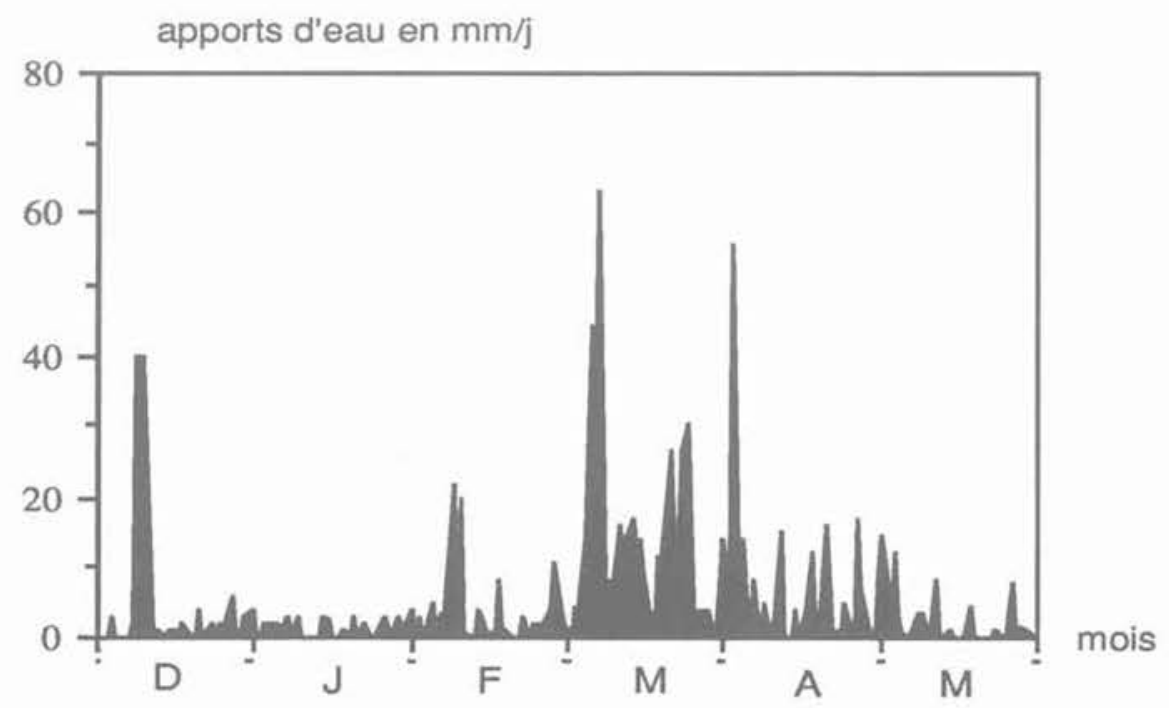

Fig. 18. - Apports d'eau au versant de La Clapière de décembre 1990 à mai 1991. Fig. 18. - Water input at La Clapière slope from december 1990 to may 1991. 


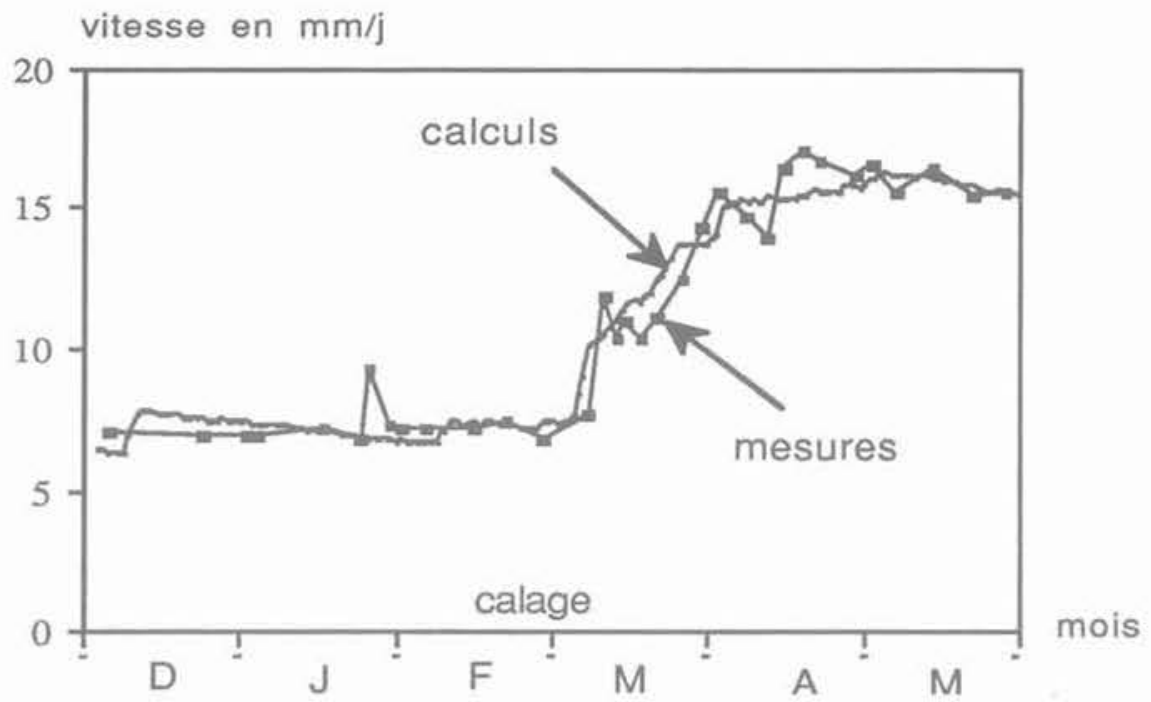

Fig. 19. - Vitesses mesurées et vitesses calculées du glissement de La Clapière de décembre 1990 à mai 1991.

Fig. 19. - Comparaison between observed and computed rate of displacement at La Clapière rate of from december 1990 to may 1991.

niveau des prévisions par une dérive progressive ; une " actualisation » du modèle doit être faite régulièrement : pour ce glissement nous avons retenu une période d'un mois.

\section{CONCLUSION}

Grâce au suivi en continu de mouvements de terrains, il est possible de modéliser les comportements de versants instables de façon relativement précise.

Nous avons vu qu'une démarche purement analytique n'est guère possible actuellement, mais une approche "pseudo-empirique », en partie appuyée sur la connaissance de phénomènes physiques connus dans les versants donne de bons résultats pour les cas étudiés.

La prévision temporelle des mouvements de versants constitue un enjeu important, car elle donne aux pouvoirs publics une information quantitative indispensable à une prise de décision lorsque des populations ou des biens sont menacés directement.

Les modèles de prévision proposés ici constituent le premier maillon du processus complet qui doit comporter une évaluation quantitative des risques générés par les mouvements des versants, et un système d'alerte aux populations.

\section{REMERCIEMENTS}

Les auteurs expriment leur reconnaissance à JeanPaul FOLLACCI et Thierry GOUIN qui leur ont communiqué des données de mesures et ont participté aux interprétations.

\section{BIBLIOGRAPHIE}

ANTOINE P. et al. (1987), Le menace d'écroulement aux ruines de Séchilienne (Isère). Bulletin de liaison des laboratoires des Ponts et Chaussées, $\mathrm{n}^{\circ} 150$, juillet-octobre, pp. 55-64.

ASAOKO A. (1978), Observational procedure of settlement prediction, Soils and foundations. vol. 18 , $n^{\circ} 4$, déc.

AZIMI C., DESVARREUX P. (1986), Etude et prévision des mouvements de terrains. XYZ, Revue de l'Association française de topographie, $n^{\circ} 28$, sept., pp. 8-14.

CORBYN J.A. (1992), Failure of a partially submerged rock slop with particular references to the Vajont rock slide. Int. J. Rock Mech. Min. Sci. \& Geomech. Abstr., vol. 19, pp. 99-102.

FOLLACCI J.-P. (1987), Les mouvements de La Clapière à Saint-Etienne-de-Tinée. Bulletin de liaison des laboratoires des Ponts et Chaussées, $\mathrm{n}^{\circ}$ 150-151, juillet-octobre, pp. 39-54.

GERVREAU E. (1991), Etude et prévision de l'évolution des versants naturels en mouvement. Coll. Etudes et recherches des laboratoires des Ponts et Chaussées, série géotechnique GT 47.

SAITO M. (1969), Forecasting time of slope failure by tertiary creep. $7 e$ congrès international de mécanique des sols et travaux de fondation, Mexico, vol. 2, pp. 677-683.

VOIGHT B. (1989), Deformation and failure-time prediction in rock mechanics. Proc. of 30 th US symposium Rock Mechanics, Morgantown, pp. 919-929. 